\title{
Low-cost lipid production by an oleaginous yeast cultured in non-sterile conditions using model waste resources
}

\author{
Fabio Santamauro ${ }^{1,2+}$, Fraeya M Whiffin ${ }^{2}$, Rod J Scott ${ }^{{ }^{* \dagger}}$ and Christopher J Chuck ${ }^{2^{*+}}$
}

\begin{abstract}
Background: The yeast Metschnikowia pulcherrima, previously utilised as a biological control agent, was evaluated for its potential to produce lipids for biofuel production.

Results: Cultivation in low cost non-sterile conditions was achieved by exploiting its ability to grow at low temperature and $\mathrm{pH}$ and to produce natural antimicrobial compounds. Although not previously classified as oleaginous, a combination of low temperature and restricted nutrient availability triggered high levels of oil production in M. pulcherrima cultures. This regime was designed to trigger the sporulation process but prevent its completion to allow the accumulation of a subset of a normally transitional, but oil-rich, 'pulcherrima' cell type. This approach resulted in yields of up to $40 \%$ lipid, which compares favourably with other oleaginous microbes. We also demonstrate that $M$. pulcherrima metabolises glycerol and a diverse range of other sugars, suggesting that heterogeneous biomass could provide a suitable carbon source. M. pulcherrima also grows well in a minimal media containing no yeast extract. Finally, we demonstrate the potential of the yeast to produce lipids inexpensively on an industrial scale by culturing the yeast in a $500 \mathrm{~L}$, open air, tank reactor without any significant contamination.
\end{abstract}

Conclusions: The production of antimicrobial compounds coupled to efficient growth at low temperature and $\mathrm{pH}$ enables culture of this oleaginous yeast in inexpensive, non-sterile conditions providing a potential route to economic biofuel production.

Keywords: Biodiesel, Lipid, Yeast, Heterotrophic, Waste, Pilot scale, Glycerol, Lignocellulose

\section{Background}

The rising cost of fossil fuels, coupled to concerns over security of supply and an increasing awareness of the environmental impact of associated $\mathrm{CO}_{2}$ emissions, have led to the development of alternative energy solutions becoming a global priority. Some of the most ambitious national targets have been set by the United Kingdom, where the government is committed to sourcing $15 \%$ of the total energy demand from renewable resources by 2020 , and $80 \%$ by 2050 [1]. Since a quarter of emissions are produced by the transport sector, meeting these reduction targets requires urgent development of biofuels compatible with the current infrastructure. Currently,

\footnotetext{
* Correspondence: R.J.Scott@bath.ac.uk; C.Chuck@bath.ac.uk

${ }^{\dagger}$ Equal contributors

${ }^{1}$ Department of Biology and Biochemistry, University of Bath, Bath BA2 7AY, UK ${ }^{2}$ Centre for Sustainable Chemical Technologies, Department of Chemical Engineering, University of Bath, Bath BA2 7AY, UK
}

the main source of biofuels is sugar-derived bioethanol or biodiesel produced from lipids, derived from oil palm, rapeseed or soybean [2]. However, these crops compete with food crops for agricultural land and consequently have both a negative public image and environmental impact [3]. Oleaginous microorganisms such as microalgae, bacteria, fungi and yeasts are an attractive alternative to higher plants for lipid production, since their production does not require agricultural land, avoiding displacement of food production. While species from these taxa have the potential to produce lipids, only microalgae have been extensively investigated to date [4]. Although algae have great theoretical potential as a fuel source, substantial technical hurdles currently prevent their cost-effective exploitation. These include the scarcity of suitable land, given the current levels of productivity, the lack of adequate photosynthetically active radiation and the prohibitive costs of delivering supplementary

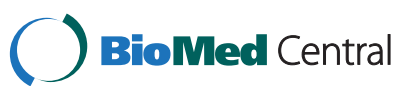


light, fertiliser, controlling temperature and critically, protecting against invasive non-lipid-producing species $[5,6]$.

Heterotrophic organisms, such as yeasts, offer a credible alternative to microalgae for the production of biofuel feedstocks, especially in Northern Europe. Oleaginous yeast species are highly productive on a per-cell basis, with lipid yields of up to $65 \%$ dry weight and grow to high densities with biomass yields of 10 to $100 \mathrm{~g} \cdot \mathrm{L}^{-1}$ reported over 3 to 7 days [7]. In comparison, microalgae achieve only 0.15 to $0.25 \mathrm{~g} \cdot \mathrm{L}^{-1}$ per day in open pond systems [5]. The most common yeasts so far examined for biofuel production are Rhodotorula glutinis, Yarrowia lipolytica and Lipomyces starkeyi [8-11].

Recently, the economic production of $\mathrm{C}_{5}$ and $\mathrm{C}_{6}$ sugars from waste cellulosic materials has become plausible, making plant sugars derived from lignocellulose a feasible source of renewable feedstocks $[12,13]$. Unlike microalgae, yeast cultivation does not require light, which both reduces input costs and enables production $24 \mathrm{~h}$ per day. Essential inputs such as phosphorous and nitrogen are also available from waste streams such as waste water, again reducing production costs.

One key issue with the cultivation of heterotrophic organisms is maintaining an axenic culture. Bacteria from the surrounding environment or contaminating input waste streams have the potential to outcompete most types of oleaginous yeast. Ensuring the strict sterile conditions required for production of triglycerides by yeast, therefore, requires stringent pre-treatment of the feedstock and careful enclosure of the fermentation broth. Both significantly increase production costs, potentially limiting industrial-scale lipid biofuel production [14]. One potential solution is to use an extremophile yeast that thrives at an extreme $\mathrm{pH}$, temperature or salinity that significantly reduces or prevents competing microbial growth $[15,16]$. As bacteria and other contaminating organisms can adapt to such environments, an extremophile with more than one protective mechanism is attractive for open-tank reactors.

The non-saccharomyces yeast, Metschnikowia pulcherrima, is known to severely limit the growth of other microbes in the natural environment and is consequently used commercially as a post-harvest control agent [17]. However, the ecological significance and exact mechanisms are still poorly understood but offer the potential for commercial exploitation. M. pulcherrima, also reported in literature as Candida pulcherrima (anamorph), Saccharomyces pulcherrimus, Rhodotorula pulcherrima, Torula pulcherrima, Cryptococcus castellanii and Torulopsis pulcherrima, [18] is commonly found in flower nectar and fruit surfaces, and colonises the grape must after pressing, contributing to the flavour and aroma of the wine [19]. The yeast thrives in the must despite its high acidity ( $\mathrm{pH} 3$ to 4) and sugar concentration
(>100 g/L), and resists competition from other microorganisms. Cultures of $M$. pulcherrima do not require iron or vitamins other than biotin [20,21]. M. pulcherrima is well-known for its production of the red pigment, pulcherrimin formed by irreversible binding of pulcherrimic acid and environmental iron to create a metal-organic framework. This sequestration prevents competing microbes accessing essential supplies of iron, and is the assumed basis of the use of $M$. pulcherrima as a biofungicide in post-harvest disease control [17,22,23]. $M$. pulcherrima was therefore selected for study for its potential for low-cost culture in non-sterile conditions due a combination of acidophilia and secretion of the potent anti-microbial compounds, pulcherriminic acid and 2phenylethanol [24].

During its growth on the surface of grapes $M$. pulcherrima is assumed to catabolise constituents of the plant cell-wall, as well as accessing sugars from within the grape [25]. This accords with recent studies demonstrating that $M$. pulcherrima produces a wide range of cellwall degrading enzymes; this property indicates a degree of metabolic plasticity that lends itself to industrial applications in oenology $[19,24]$.

Whilst the innate ability of $M$. pulcherrima to grow at low $\mathrm{pH}$ and temperature combined with pulcherrimin production would facilitate its culture under relatively inexpensive non-sterile conditions, previous studies have concluded that the species is not oleaginous [26]. However, when subjected to environmental stress, for example, nitrogen starvation, a subset of cells within an $M$. pulcherrima culture begin sporulation via a transitional pulcherrima cell-type that has shown some evidence of lipid accumulation $[27,28]$. We reasoned that increasing the proportion of pulcherrima cells formed in response to a suitable stimulus, coupled with a means to prevent their development into oil-poor spores, could elevate overall lipid production by an $M$. pulcherrima culture into the oleaginous range.

Here we show that manipulating the culture regime successfully increased the proportion of oil-rich pulcherrima cells and achieved a $40 \%$ overall lipid yield. We also show that the yeast metabolises glycerol and a diverse range of other sugars, suggesting that heterogeneous biomass could provide a suitable carbon source. Finally, we illustrate the potential to produce lipids inexpensively on an industrial scale by culturing $M$. pulcherrima in a 500-L, open air, tank reactor.

\section{Results and discussion}

As a non-saccharomyces yeast involved in the first stages of wine fermentation, $M$. pulcherrima endures particularly stressful conditions in the grape must, such as high acidity due to the high levels of tartaric and malic acid, and high osmotic pressure due to the high 
sugar concentrations. The ability to thrive under such harsh conditions makes $M$. pulcherrima a potentially attractive organism for industrial applications, particularly where culture in non-sterile conditions would reduce production costs. Whilst the robustness of $M$. pulcherrima has been established, its status as an oleaginous yeast has not. We set out to determine whether M. pulcherrima is capable of high lipid-production in conditions that exploit its potential for low cost culture.

\section{Triggering oil production in $M$. pulcherrima}

Lipid accumulation in microbial cells normally requires a starvation step to promote energy storage at the expense of further cell division [29]. To achieve this, the starting concentration of nitrogen in the culture medium is deliberately limited relative to the carbon source. A recent study evaluating the potential of yeasts to produce biofuels from glycerol found that $M$. pulcherrima did not accumulate lipids to any significant degree at $28^{\circ} \mathrm{C}$, over 5 days, even at high $\mathrm{C} / \mathrm{N}$ ratios [26]. However, other types of stress induce $M$. pulcherrima to undergo sporulation via a transitory pulcherrima cell-type that accumulates high lipid levels, particularly in dilute media or at low temperatures, though the effectiveness of these treatments has not been quantified $[27,28]$. Although pulcherrima cells have not been observed as the predominant cell-type in a culture, it seemed likely that conditions of nutrient starvation and below-ambient temperatures would act as a trigger for this stage. Such low temperatures would potentially halt sporulation, resulting in increased levels of lipid-containing cells. To determine whether temperature could trigger oil production in M. pulcherrima, the culture was grown at $20^{\circ} \mathrm{C}$ for 3 days, the temperature was then modified (increased or decreased) for the remaining length of the culture period. The lipid content of the biomass was measured for the cultures after 15 days.

At $25^{\circ} \mathrm{C}, 14 \%$ lipid was extracted from the cultures, which is approaching the oleaginous range (Figure 1). As predicted, the highest lipid productivity was observed at $15^{\circ} \mathrm{C}$, with per-cell lipid content of $26 \%$. Although we did not attempt to quantify the proportion of pulcherrima in these cultures, the dramatic increase in overall lipid content suggests a substantial enrichment had been achieved for the reason outlined above. Significantly, $M$. pulcherrima grew extremely well between $15^{\circ} \mathrm{C}$ and $20^{\circ} \mathrm{C}$, reaching values of dry biomass around $6 \mathrm{~g} / \mathrm{L}$. When the stationary phase was held at $25^{\circ} \mathrm{C}$ a $20 \%$ reduction in dry biomass was observed, though there was little difference in the lipid production of the system compared to $20^{\circ} \mathrm{C}$. Not only are these temperatures too low for most common bacteria but are also ideal for producing fuels in Northern Europe without high energy-costs for heating.
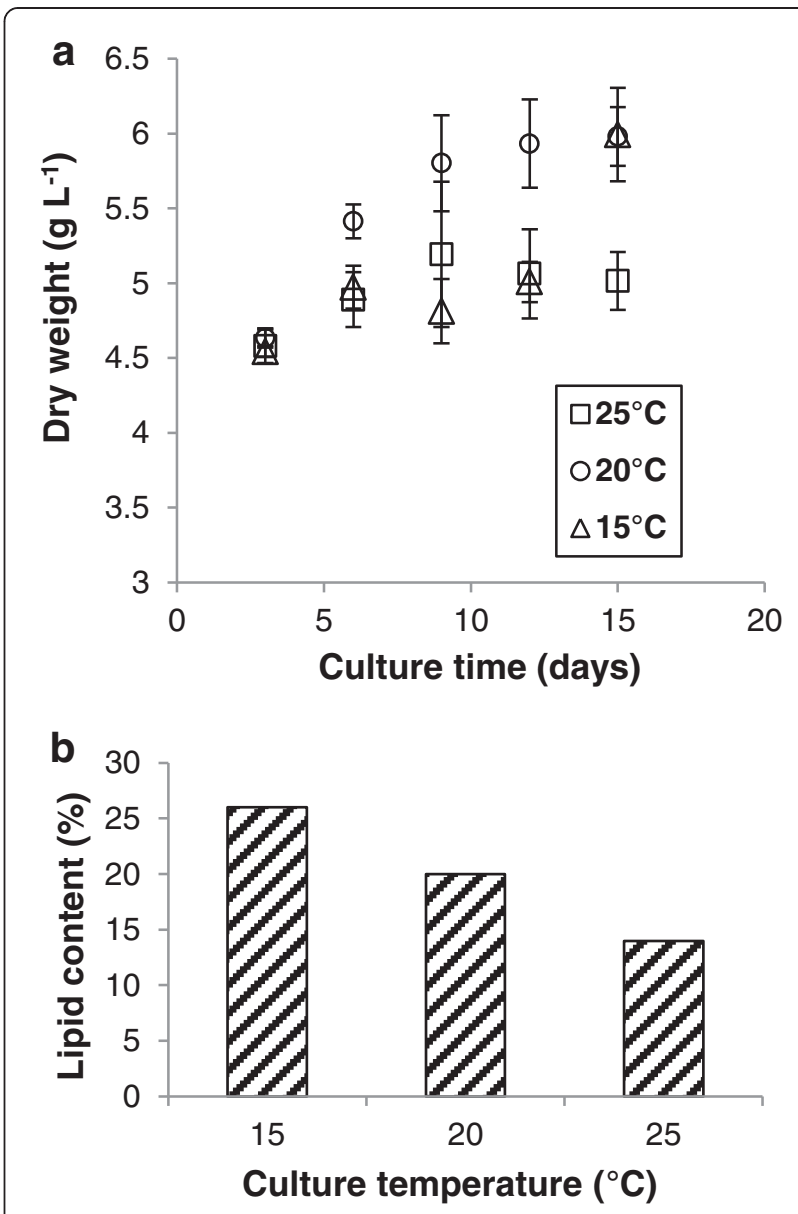

Figure 1 Effect of temperature on the growth of $M$. pulcherrima grown on standard M. pulcherrima (SMP) media (a) and total lipid content as a percentage of dry weight after 15 days growth (b).

The effect of restricted nutrients on the growth

One of the main factors in maintaining a monoculture is the ability of $M$. pulcherrima to grow at low $\mathrm{pH}$. In the literature, $M$. pulcherrima is reported to grow optimally at $\mathrm{pH}$ between 5.0 and 7.5, but has been shown to grow at $\mathrm{pH}$ as low as $3.0[22,30]$. Under the conditions used in this study M. pulcherrima grew well between $\mathrm{pH} 3.0$ and $\mathrm{pH} 6.0$, though the maximum biomass was observed at an initial $\mathrm{pH}$ of 5.0 (Figure 2a). To ensure that the conditions were capable of supporting significant lipid accumulation, a semi-quantitative assay was used. For this samples were collected at regular intervals, stained with the fluorescent dye Bodipy 493/503 and analysed by flow cytometry. Biomass production was only reduced slightly at $\mathrm{pH} 4.0$. Even at $\mathrm{pH} 3.0$, around $85 \%$ of the biomass was produced compared to that at $\mathrm{pH}$ 5.0. Irrespective of the starting $\mathrm{pH}$, or change in $\mathrm{pH}$ during the culture period, biomass productivity remained above $5 \mathrm{~g} / \mathrm{L}$. Irrespective of the starting $\mathrm{pH}, M$. pulcherrima acidified the environment, resulting in a $\mathrm{pH}$ slightly 

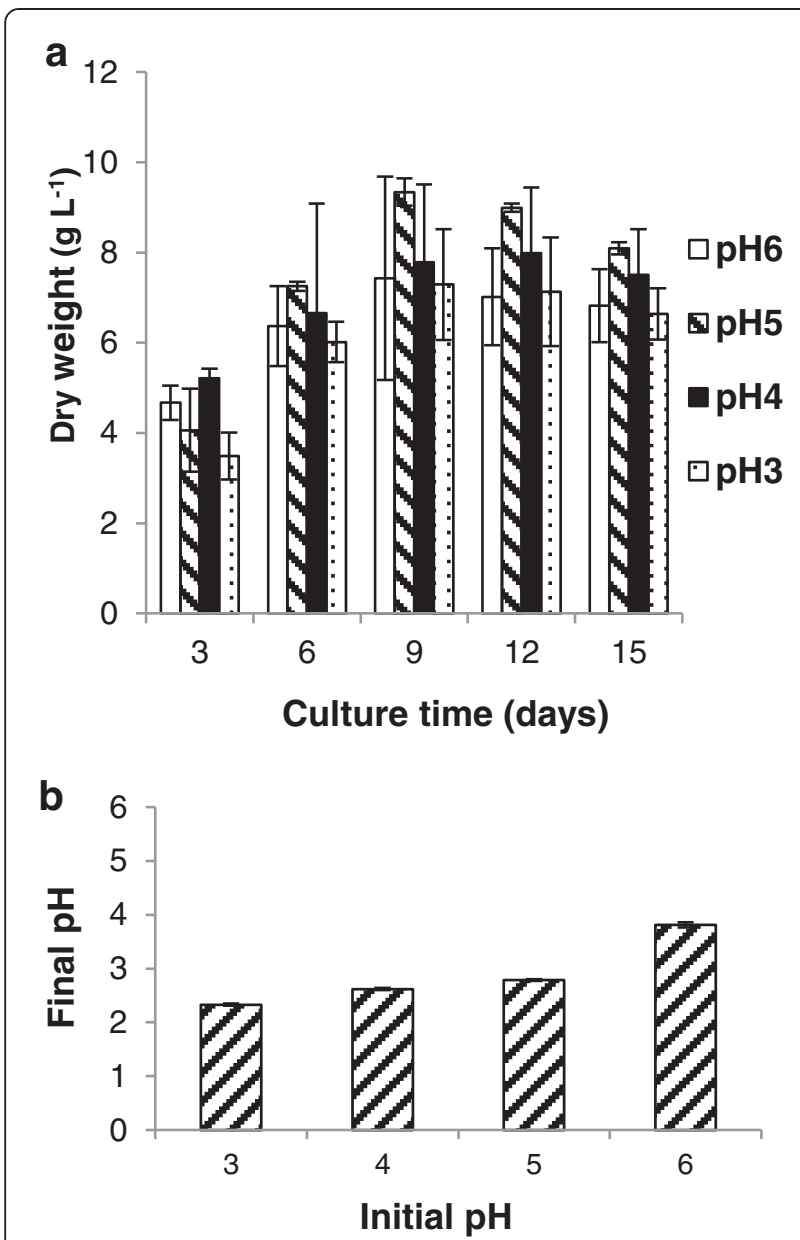

Figure 2 Effect of the initial $\mathrm{pH}$ on the growth of the $M$. pulcherrima culture grown at $20^{\circ} \mathrm{C}$ over 15 days (a) and the final $\mathrm{pH}$ of the cultures (b).

above 2 (Figure $2 \mathrm{~b}$ ). While presumably too low for optimal biomass production, this would further insure that the cultures remain sterile and that no bacteria could outcompete the yeast.

The original screening, to promote lipid accumulation in M. pulcherrima utilised glycerol as a feedstock [26].
Glycerol is an important waste product derived from the biodiesel production process as an aqueous solution (approximately $65 \% \mathrm{wt} / \mathrm{wt}$ ) contaminated with small amounts (below $5 \% \mathrm{wt} / \mathrm{wt}$ of each contaminant) of compounds such as methanol, catalyst and organic compounds. These contaminants have not been found to significantly affect the growth of other microorganisms compared to pure glycerol [31]. We therefore first tested the ability of $M$. pulcherrima to exclusively utilize $95 \%$ pure glycerol, a model waste resource, across a broad range of concentrations (1 to 25\%). The biomass content increased steadily over the 15-day culture period irrespective of the glycerol concentration (Figure 3). The maximum biomass yield was achieved with a $9 \%$ glycerol concentration, which decreased only slightly at higher concentrations. Although we did not investigate the cause of this reduction, high glycerol content could inhibit oxygen uptake or create high osmotic pressures sufficient to inhibit culture growth. However, this effect was minor and our data therefore demonstrate that the yeast thrives on a wide range of glycerol concentrations. Significantly, biomass productivity was above $5 \mathrm{~g} / \mathrm{L}$ and constant at glycerol concentrations between 5 and $9 \%$, indicating that there is little benefit in biomass terms from almost doubling the glycerol concentration.

Several other Candida species (Candida curvata, Rhodotorula graminis, Cryptococcus curvatus and Yarrowia lipolytica) reportedly produce between 28 and 53\% lipid when grown on glycerol as a carbon source. This level is comparable to that produced by the $M$. pulcherrima strain presented in this work [32,33]. M. pulcherrima grew at high glycerol concentrations, and although this has been reported for some oleaginous species such as $Y$. lipolytica, it is far from common. For example, other similar oleaginous species, such as $C$. curvatus are unable to grow on concentrations higher than $60 \mathrm{~g} / \mathrm{L}$, presumably due to the elevated osmotic pressure [31-33].

A key issue when using waste feedstocks is the potential heterogeneity of supply, which demands that the cultured organism possesses a degree of flexibility in its

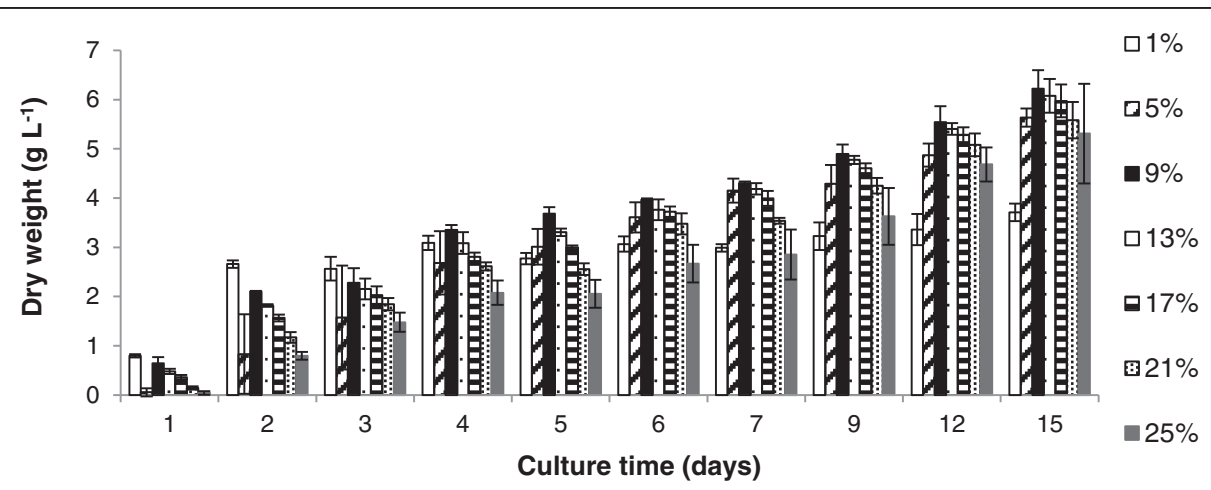

Figure 3 Effect of glycerol concentration on the growth of a culture of $M$. pulcherrima, grown at $20^{\circ} \mathrm{C}$ over 15 days with a starting pH of 5. 
nutrient and environmental requirements. To assess $M$. pulcherrima for its potential for robust waste feedstock utilisation we measured the impact of different nutrient sources on growth dynamics. Three alternative nitrogen sources $\left(\mathrm{NH}_{4} \mathrm{Cl}, \mathrm{NH}_{4} \mathrm{NO}_{3}\right.$ and $\left.\mathrm{Ca}\left(\mathrm{NO}_{3}\right)_{2}\right)$ were evaluated by replacing $\left(\mathrm{NH}_{4}\right)_{2} \mathrm{SO}_{4}$ in the standard $M$. pulcherrima (SMP) containing $10 \% \mathrm{w} / \mathrm{v}$ glycerol. The total nitrogen concentration was kept constant when using the different nitrogen sources. Biomass productivity was greatest using ammonium salts compared to nitrates (Figure 4a), with $\mathrm{NH}_{4} \mathrm{Cl}$ supporting a growth rate comparable to $\left(\mathrm{NH}_{4}\right)_{2} \mathrm{SO}_{4}$.
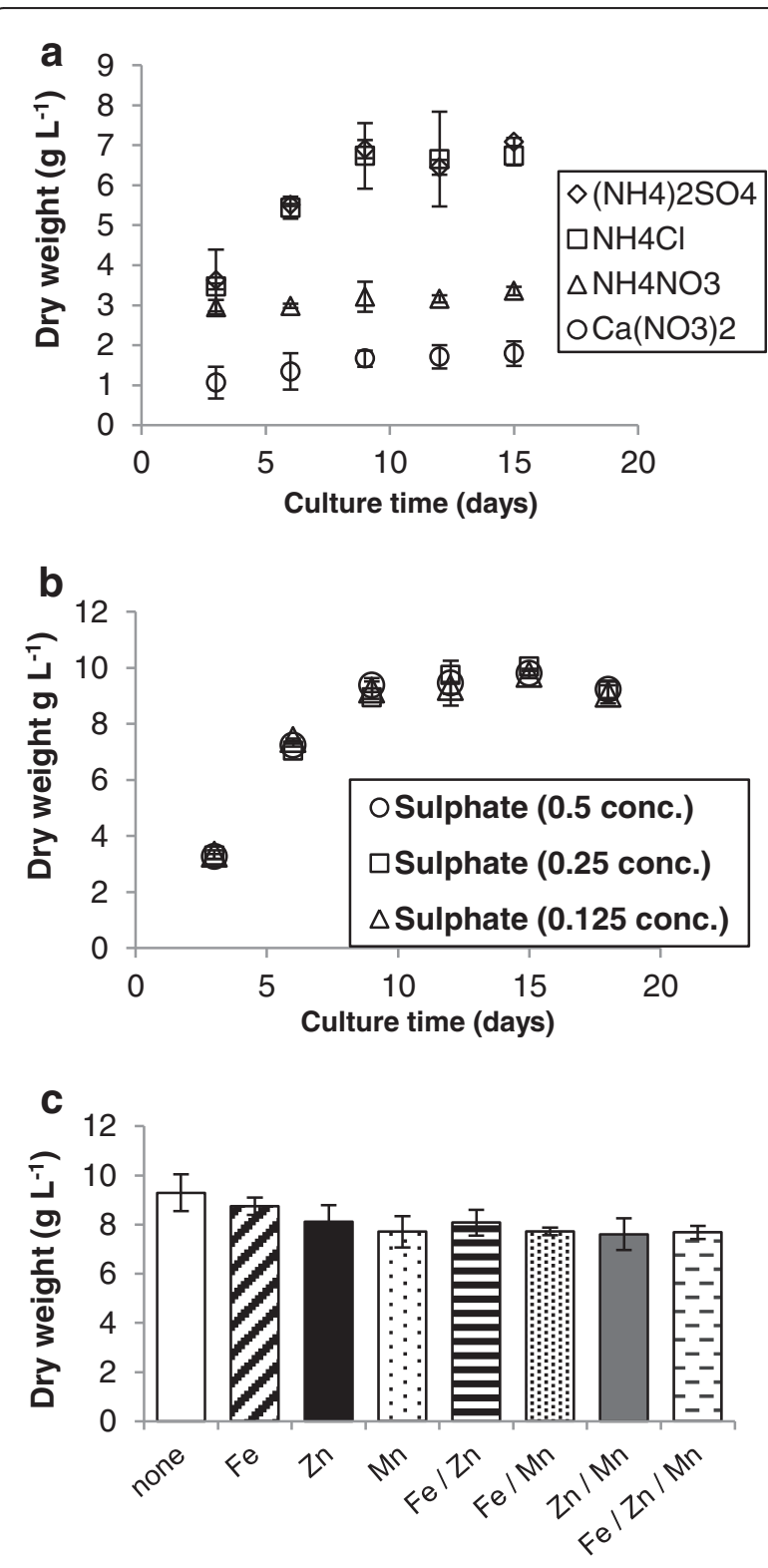

Nutrient removed from the culture

Figure 4 Nutrient requirements for $M$. pulcherrima cultivation on glycerol. a) Nitrogen source, b) sulphur content and c) demonstrates the removal of micronutrients.
In contrast, the performance of $\mathrm{NH}_{4} \mathrm{NO}_{3}$ and $\mathrm{Ca}\left(\mathrm{NO}_{3}\right)_{2}$ was below $3 \mathrm{~g} / \mathrm{L}$ indicating that these compounds are not suitable nitrogen sources for $M$. pulcherrima.

Sulphur is another key nutrient that can affect growth rates. Generally sulphur is present as sulphates, especially in waste water. To assess the flexibility of $M$. pulcherrima, a range of cultures were examined with reduced levels of sulphate (Figure 4b). There was no difference, in terms of dry biomass; on culturing $M$. pulcherrima with concentrations of sulphur between $0.321 \mathrm{~g} / \mathrm{L}$ and $0.045 \mathrm{~g} / \mathrm{L}$, the same level of biomass were obtained.

A range of micronutrients is added to most yeast culture media, including salts of manganese, zinc and iron. Although these micronutrients are potentially present in waste water, the type and amount of the micronutrient will vary substantially [34]. To determine the tolerance of $M$. pulcherrima to fluctuations in micronutrient availability, the yeast was grown in SMP (10\% w/v glycerol) lacking $\mathrm{Fe}, \mathrm{Zn}$, or $\mathrm{Mn}$, either singly or in combination. The biomass production after 15 days was then compared to a culture grown on SMP with all the nutrients present (Figure 4c). Surprisingly, biomass productivity was reduced by only $15 \%$ in SMP not supplemented with iron, zinc and manganese.

M. pulcherrima demonstrates excellent flexibility on being cultured with different types and amounts of nitrogen, sulphur and micronutrients. This demonstrates that although there is an optimal culture configuration that produces the highest levels of biomass from any given system, the effect of removing key nutrients does not dramatically reduce biomass yields. M. pulcherrima, therefore, has excellent adaptability to any changes in the waste water or alternative nutrient streams that could be used to culture the system.

Following these promising results we modified SMP to optimise lipid accumulation. The new medium, optimised M. pulcherrima (OMP) medium lacked manganese and iron, and had lower levels of sulphur. Cultures grown in this medium achieved higher lipid content whilst retaining biomass yields of around $7 \mathrm{~g} / \mathrm{L}$. In a typical experiment $M$. pulcherrima was grown in $\mathrm{OMP}$ at $25^{\circ} \mathrm{C}$ for the first 3 days, than at $15^{\circ} \mathrm{C}$ for a further 12 days. After 15 days a $7.4 \mathrm{~g} \cdot \mathrm{L}^{-1}$ biomass yield was achieved, which had a total lipid content of $40 \%$ (Figure 5). This demonstrates that with an appropriate combination of medium and temperature regime, the oil productivity of $M$. pulcherrima is well into the oleaginous range.

Lipid accumulation in $M$. pulcherrima continues up to 15 days, after which the lipid content decreases, presumably due to cellular lipid turnover (Figure 5). This phenomenon has been reported for the yeast $Y$. lipolytica and other oleaginous fungi, for example, Cunninghamella echinulata [35]. The authors propose that the lipid degradation occurs in C. echinulata by selective use 


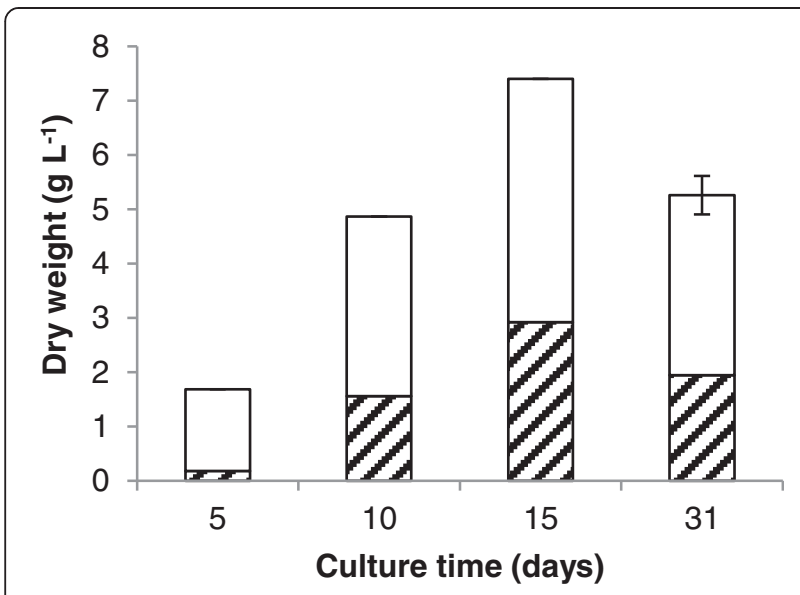

Figure $\mathbf{5}$ Total biomass and lipid produced (shaded area) using an optimised media.

of triglyceride to produce glycolipids, sphingolipids and phospholipids through the substitution of one or more fatty acid (FA) chains. The triglyceride can also be completely hydrolysed to glycerol and free fatty acids, which can then be funnelled into glycolysis and $\beta$-oxidation.

\section{Alternative feedstocks}

Although $M$. pulcherrima is productive using a glycerol carbon source, a far more abundant feedstock is lignocellulose. Waste food, agricultural wastes or energy crops grown specifically for the purpose can be converted into a range of sugars through relatively inexpensive chemical or enzymatic processes [36]. The composition of the sugars obtained depends heavily on the method and source of the feedstock, and the main sugars are glucose, arabinose, xylose and cellobiose, and a range of oligosaccharides [37]. To establish its ability to utilise depolymerised lignocellulose, M. pulcherrima was cultivated on a wide range of sugars including lactose and sucrose (Figure 6). Biomass productivity for $M$. pulcherrima was higher in media containing glucose compared to any other sugar or combination of sugars. Provided glucose was present, any other sugar acted as an effective carbon source with little reduction in biomass yield. A possible explanation is that glucose induces $M$. pulcherrima to express enzymes capable of metabolising oligosaccharides, as occurs in a range of non-saccharomyces yeast [38]. Although $M$. pulcherrima was capable of growth on xylose, this resulted in reduced biomass productivity compared to glucose. M. pulcherrima struggles to grow on the arabinose aldehyde and lactose but metabolises sucrose extremely effectively, obtaining dry biomass content similar to glucose. Overall, $M$. pulcherrima can be cultivated effectively on a range of sugars, especially in the presence of glucose, indicating its suitability to utilize abundant biomass sources, such as lignocellulose.

As the main products of the depolymerisation of lignocellulose are glucose, arabinose, xylose and cellobiose, $M$. pulcherrima was cultured on a mixture of these sugars as a model waste resource and the biomass and lipid productivity established (Figure 7). M. pulcherrima produced amounts of biomass and lipid on this feedstock similar to levels achieved on glycerol. Significantly, with the exception of xylose, all four sugars of the model feedstock were starting to be catabolised by $M$. pulcherrima within the first 24 hours. Xylose consumption commenced within 48 hours. By 72 hours the culture had entered the stationary phase (data not shown), and the consumption of glucose, arabinose and cellobiose practically halted. Interestingly, xylose was continually consumed until exhausted after 12 days. It is possible that $M$. pulcherrima catabolises xylose directly, or that xylose is converted into an unknown secondary product. On exhaustion of the xylose, M. pulcherrima resumed using other sugars in the broth. After 15 days, both the glycerol and model lignocellulose cultures, had consumed nearly $20 \mathrm{~g} \cdot \mathrm{L}^{-1}$ of sugar, which was two thirds of the original starting culture.

To increase the cell density, separate cultures were investigated under a fed-batch regime, using equal amounts of the four sugars and varying the amount of yeast extract, biotin and additional nitrogen (Table 1, Figure 8). To verify whether $M$. pulcherrima was capable of contaminationfree growth in a fed-batch system the yeast was cultured in media made up in final effluent waste water. None of the

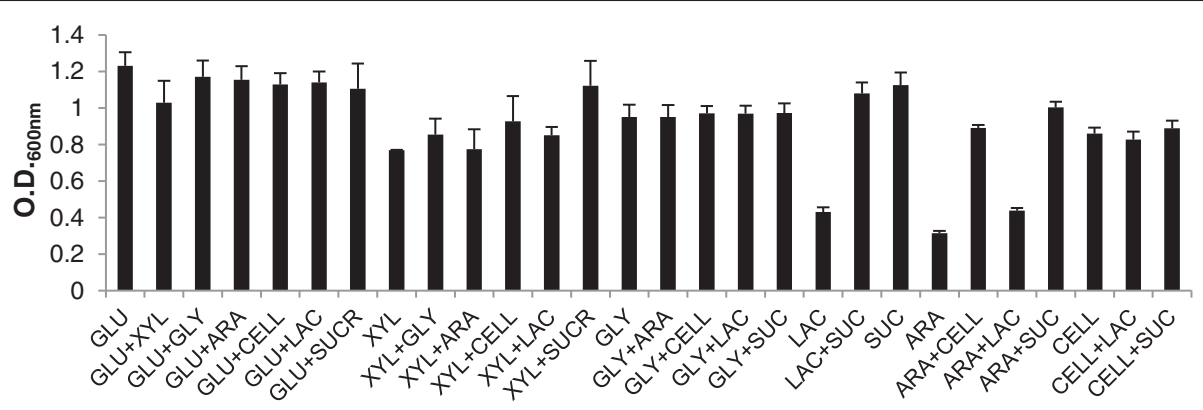

Figure 6 Biomass productivity of $\boldsymbol{M}$. pulcherrima on a range of simple sugars. Optical density (O.D.) of the culture after 15 days. GLU, glucose; XYL, xylose; GLY, glycerol; ARA, arabinose; CELL, cellobiose; LAC, lactose; SUC, sucrose. 


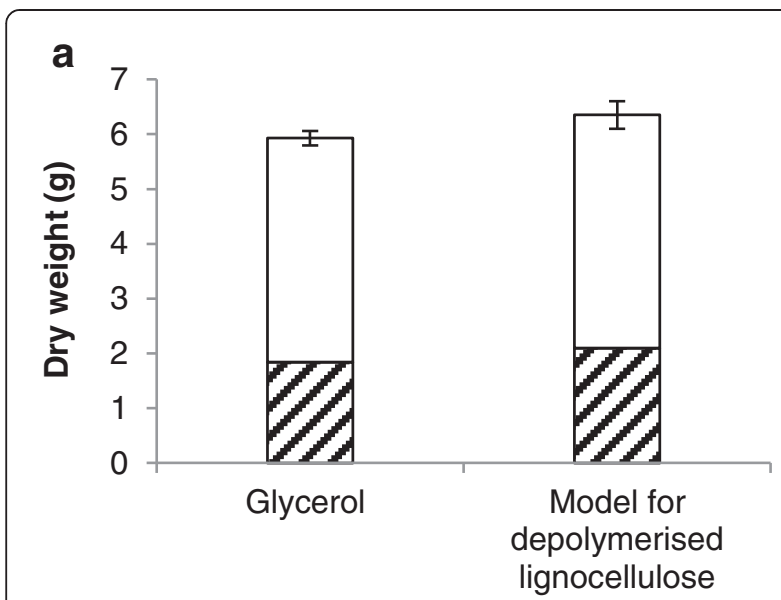

b

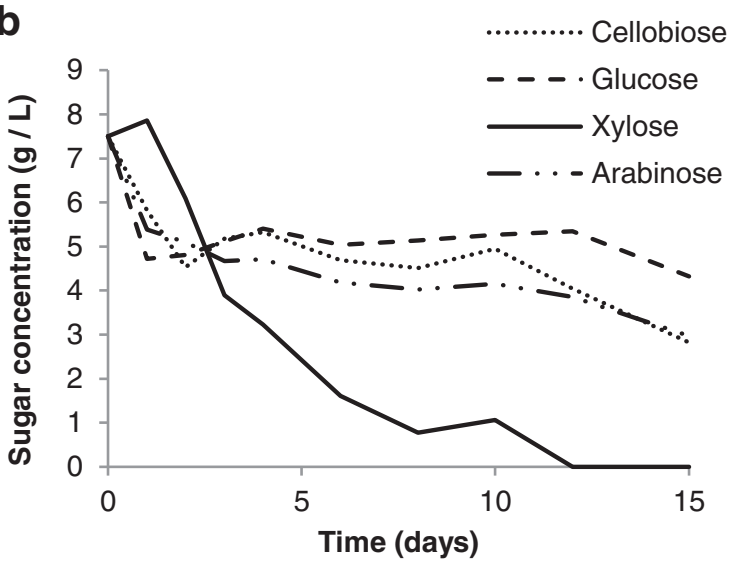

C

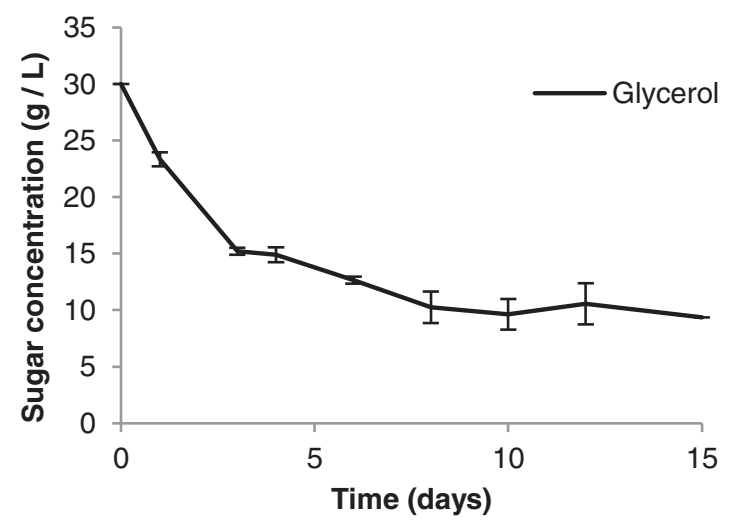

Figure 7 Total biomass and lipid produced (shaded area) for glycerol and a model for depolymerised lignocellulose in optimised media, and consumption of sugars and glycerol over the culture period. a) Total biomass and lipid produced and model for depolymerised lignocelluloses. The model contained $7.5 \mathrm{~g}$, arabinose, $7.5 \mathrm{~g}$ xylose, $7.5 \mathrm{~g}$ glucose and $7.5 \mathrm{~g}$ cellobiose as the carbon source. b) Consumption of sugars. c) The consumption of glycerol over the culture period.
Table 1 Lipid profile of fed-batch cultures of M. pulcherrima

\begin{tabular}{|c|c|c|c|c|}
\hline & $\begin{array}{c}\text { Run } 1 \\
\text { (with yeast } \\
\text { extract) }\end{array}$ & $\begin{array}{c}\text { Run } 2 \\
\text { (without } \\
\text { yeast extract) }\end{array}$ & $\begin{array}{l}\text { Run } 3 \\
\text { (with } \\
\text { biotin) }\end{array}$ & $\begin{array}{c}\text { Run } 4 \\
\text { (without } \\
\text { biotin) }\end{array}$ \\
\hline Yeast extract & + & - & - & - \\
\hline Biotin & - & + & + & - \\
\hline Additional $\mathrm{NH} 4 \mathrm{Cl}$ & - & + & - & - \\
\hline $\begin{array}{l}\text { Final biomass } \\
\text { yield }\left(g \cdot L^{-1}\right)\end{array}$ & 17.3 & 14.6 & 14.3 & 13.4 \\
\hline $\begin{array}{l}\text { Final lipid content } \\
\text { (\% dry weight) }\end{array}$ & 35 & 39 & 19 & 33 \\
\hline
\end{tabular}

$+=$ nutrient included, $-=$ nutrient absent.

reagents were sterilised prior to use. Each culture was fed every 2 to 3 days up to 24 days $\left(25^{\circ} \mathrm{C}, 180 \mathrm{rpm}\right)$ and then allowed to rest at $15^{\circ} \mathrm{C}$ for 12 days without agitation. Other than yeast extract, biotin and the carbon source all nutrients were supplied according to the OMP media, except phosphate, which was supplemented only at 15 days since the starting concentration high excess compared to the nutritional requirement on the yeast.

M. pulcherrima was successfully grown in a fed-batch culture, with over $17 \mathrm{~g} \cdot \mathrm{L}^{-1}$ biomass being produced over 30 days. Unsurprisingly, the highest biomass yields were achieved with yeast extract, though this is not essential to growth as comparable productivity was observed for all cultures irrespective of the supplementary nutrients. The highest lipid content of $39 \%$, was produced by the culture without yeast extract but with a supplementation of biotin and nitrogen. Surprisingly, dry biomass productivity around $13 \mathrm{~g} / \mathrm{L}$ and lipid productivity around $33 \%$ were still observed in the cultures containing no yeast extract, biotin or additional nitrogen. The ability to

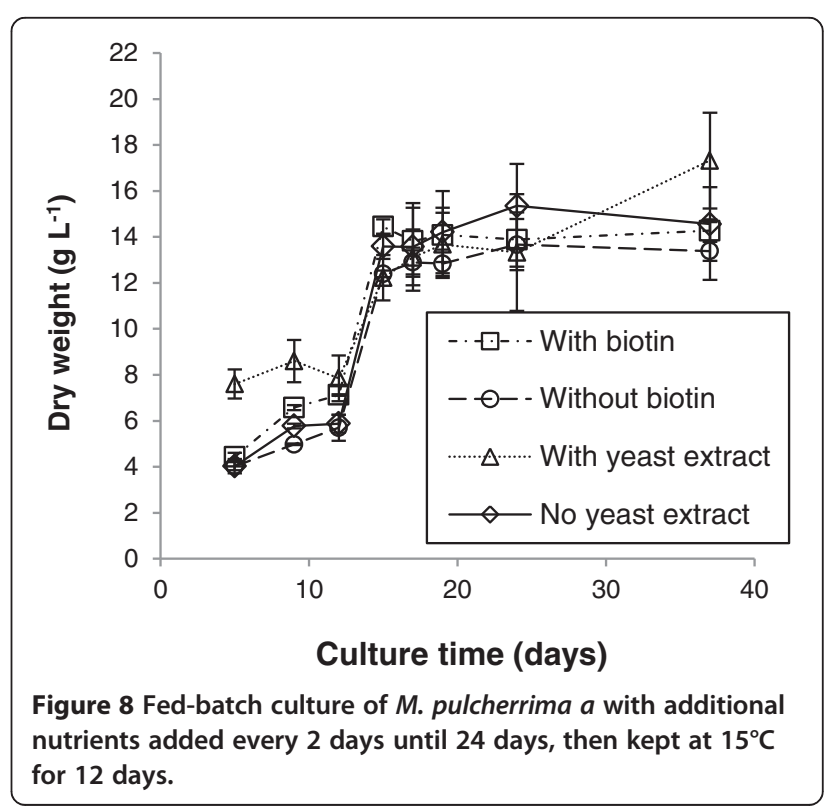


produce lipids from a complex sugar feed, with no additional yeast extract or vitamin supplementation, has significant ramifications for the viability of any future industrial process.

\section{Open-air stirred tank production}

To demonstrate the suitability of $M$. pulcherrima for low-cost production, it was grown in a hostile environment with limited temperature control on a model waste resource. The yeast was cultured in a glycerol-based OMP media in non-sterile conditions in two 500-L raceway ponds situated in a temperature-controlled glasshouse (Figure 9). The ponds were gently agitated by a paddle wheel at $10 \mathrm{rpm}$ and maintained for 15 days (Figure 10). The temperature remained roughly constant at $21^{\circ} \mathrm{C}$ irrespective of the conditions outside the glasshouse. During culture, M. pulcherrima was expected to regulate the $\mathrm{pH}$ of the medium by producing both acids and bases depending on the stage of the growth cycle. To maintain a healthy population of $M$. pulcherrima, while retaining acceptable rates of lipid and biomass production, $\mathrm{pH}$ was artificially held between 3 and 4 by the addition of weak solutions of either $\mathrm{HCl}$ or $\mathrm{KOH}$. Although some bacteria were observed at various times over the first 72 hours of culture, the population remained overwhelmingly $M$. pulcherrima (as judged by flow cytometry; data not shown). After 2 weeks when the $\mathrm{pH}$ was manually reduced to 3 , no further contamination was observed for the remainder of the culture period. When the cultures in the raceway ponds reached a value of absorbance at $600 \mathrm{~nm}$ of around 10, many large $M$. pulcherrima colonies were observed growing on the paddle wheels. These colonies developed an intense pink colour compared to the suspended culture, presumably due to increased pulcherrimin production associated with elevated levels of oxygenation [20]. Over the culture period, biomass accumulation measured by flow cytometry increased steadily reaching a final value of $2.06 \mathrm{~g} \cdot \mathrm{L}^{-1}$ (Table 2).

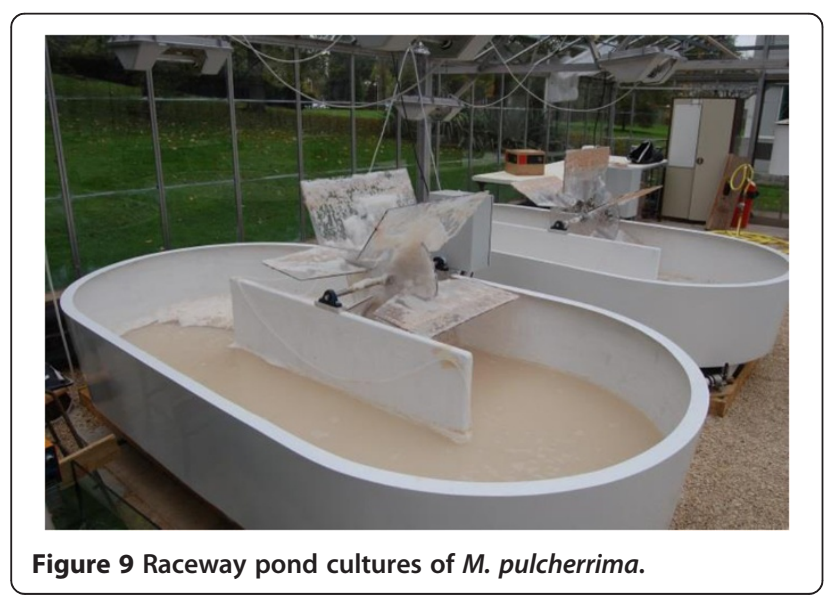

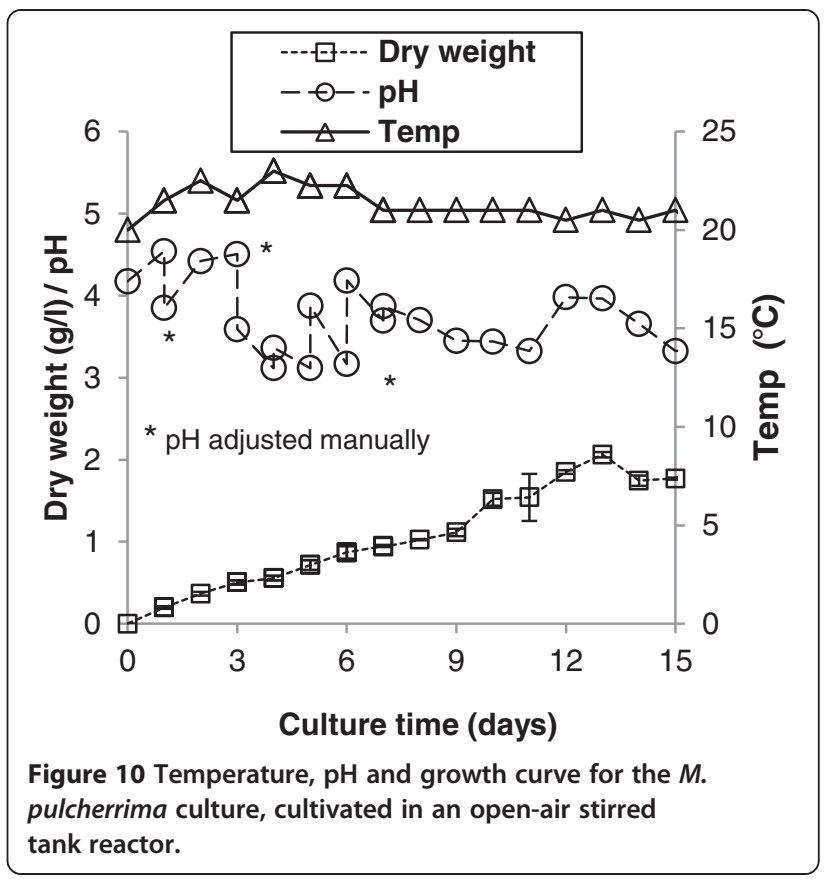

Although total biomass yield was lower than obtained at laboratory scale, these suboptimal conditions nevertheless outperformed similar systems used to culture microalgae [39]. Alongside sterilisation, agitation is a major cost in running an industrial bioprocess [13]. Though further work is needed to optimise cultures of $M$. pulcherrima on this scale, the preliminary findings demonstrate that the yeast can be cultured under nonsterile conditions, with minimal agitation. This has the potential to substantially reduce the costs of a process.

Table 2 Biomass characteristics and lipid profile of the oil produced from $M$. pulcherrima grown in the raceway ponds

\begin{tabular}{lcc}
\hline Component & & Value \\
\hline Biomass & & $2.06 \mathrm{~g} \cdot \mathrm{L}^{-1}$ \\
Lipid & & $34 \mathrm{wt} \%$ \\
Sterol & $\mathbf{1 4 : 0}$ & $18 \mathrm{~mol} \%$ \\
Fatty acids & $\mathbf{1 5 : 0}$ & $<1 \%$ \\
& $\mathbf{1 6 : 0}$ & $<1 \%$ \\
& $\mathbf{1 6 : 1}$ & $21 \%$ \\
& $\mathbf{1 7 : 0}$ & $8 \%$ \\
& $\mathbf{1 8 : 0}$ & $1 \%$ \\
& $\mathbf{1 8 : 1}$ & $4 \%$ \\
& $\mathbf{1 8 : 2}$ & $50 \%$ \\
& $\mathbf{1 8 : 3}$ & $11 \%$ \\
& $\mathbf{1 9 : 0}$ & $7 \%$ \\
& $\mathbf{2 1 : 0}$ & $<1 \%$ \\
& & $<1 \%$ \\
\hline
\end{tabular}


The level of oil extracted from the harvested biomass was $34 \%$ of the dry weight.

\section{Conclusions}

The biological control yeast, $M$. pulcherrima, which was previously classified as non-oleaginous, is shown here capable of producing up to $40 \%$ lipid by manipulating $\mathrm{pH}$ and temperature. The yeast also uses a variety of sugars potentially sourced from waste lignocellulose. Fed-batch culture using these sugars achieved a total biomass yield of $17 \mathrm{~g} \cdot \mathrm{L}^{-1}$. High biomass productivity was also achieved using simplified media lacking expensive inputs such as yeast extract and biotin. The unique combination of acidophilia and production of antimicrobials enable culture in lost-cost non-sterile conditions providing a potentially economically viable biofuel production platform.

\section{Methods}

\section{Materials}

All chemicals were purchased from Sigma Aldrich unless otherwise stated and used without purification.

\section{Culture conditions on a laboratory scale}

Cultures were grown from a single colony taken from a yeast extract, malt extract and dextrose (YMD) agar plate suspended in $10 \mathrm{ml}$ YMD (yeast extract $10 \mathrm{~g} \cdot \mathrm{L}^{-1}$; malt extract $20 \mathrm{~g} \cdot \mathrm{L}^{-1}$; glucose $\left.20 \mathrm{~g} \cdot \mathrm{L}^{-1}\right)$ and inoculated into liquid standard $M$. pulcherrima (SMP) medium adapted from Chatzifragkou et al. $\left(\mathrm{KH}_{2} \mathrm{PO}_{4} 7 \mathrm{~g} \cdot \mathrm{L}^{-1} ; \mathrm{Na}_{2} \mathrm{HPO}_{4} 2.5 \mathrm{~g} \cdot \mathrm{L}^{-1}\right.$; $\mathrm{MgSO}_{4} \cdot 7 \mathrm{H}_{2} \mathrm{O} 1.5 \mathrm{~g} \cdot \mathrm{L}^{-1} ; \mathrm{CaCl}_{2} \cdot 2 \mathrm{H}_{2} \mathrm{O} 0.15 \mathrm{~g} \cdot \mathrm{L}^{-1} ; \mathrm{ZnSO}_{4}$ $7 \mathrm{H}_{2} \mathrm{O} 0.02 \mathrm{~g} \cdot \mathrm{L}^{-1} ; \mathrm{MnSO} 4 \cdot \mathrm{H}_{2} \mathrm{O} 0.06 \mathrm{~g} \cdot \mathrm{L}^{-1} ; \mathrm{FeCl}_{3} 0.15 \mathrm{~g} \cdot \mathrm{L}^{-1}$; $\left(\mathrm{NH}_{4}\right)_{2} \mathrm{SO}_{4} 0.5 \mathrm{~g} \cdot \mathrm{L}^{-1}$ and yeast extract $\left.1 \mathrm{~g} \cdot \mathrm{L}^{-1}\right)$ [23]. The medium was autoclaved for 20 minutes at $120^{\circ} \mathrm{C}$ prior to use. Each experiment was conducted in triplicate using $10 \mathrm{ml} \mathrm{SMP}$ medium in 50-ml Falcon tubes.

The optimum glycerol concentration was determined using SMP medium containing between 1\% and 25\% $(\mathrm{w} / \mathrm{v})$ glycerol. To examine the effect of temperature on lipid production, cultures were grown for 3 days at $25^{\circ} \mathrm{C}$ then switched to either $15^{\circ} \mathrm{C}$ or $20^{\circ} \mathrm{C}$ for the remaining period. Alternative nitrogen sources were evaluated by replacing $\left(\mathrm{NH}_{4}\right)_{2} \mathrm{SO}_{4}$ in SMP medium (10\% w/v glycerol) and maintaining the total nitrogen concentration at $0.106 \mathrm{~g} \cdot \mathrm{L}^{-1}$. Micronutrient deficiency was investigated using SMP medium (10\% w/v glycerol) lacking the specified micronutrient. Sulphur content was varied using modified SMP medium (10\% w/v glycerol) containing 50\%, 25\% and $12.5 \%$ of the concentration in SMP media. $\mathrm{pH}$ was adjusted using dilute $\mathrm{HCl}$ or $\mathrm{KOH}$ to a achieve a range between $\mathrm{pH} 3$ and $\mathrm{pH}$ 6. Each set of experiments were conducted over 15 days. Optical density (O.D.) $600 \mathrm{~nm}$, cell number and lipid fluorescence were measured at 72-hour intervals.

\section{Alternative sugar sources}

The performance of $M$. pulcherrima on different sugar sources (glucose, glycerol, xylose, arabinose, cellobiose, lactose, sucrose and glycerol) was examined using a 96well microtitre plate system (Versamax, Molecular devices, UK). Briefly, $200 \mu \mathrm{l}$ of culture were made up in 96-well plates in SMP medium containing a $30 \mathrm{~g} \cdot \mathrm{L}^{-1}$ of the sugar. After 15 days at $25^{\circ} \mathrm{C}, 180 \mathrm{rpm}$, the O.D.600nm was measured using a plate reader. All the possible combinations of two sugars were tested using six repeats for each combination. An OMP medium was developed for low-cost culture of $M$. pulcherrima. OMP consisted of: $\mathrm{KH}_{2} \mathrm{PO}_{4} 7 \mathrm{~g} \cdot \mathrm{L}^{-1} ; \mathrm{Na}_{2} \mathrm{HPO}_{4} 2.5 \mathrm{~g} \cdot \mathrm{L}^{-1} ; \mathrm{MgSO}_{4} \cdot 7 \mathrm{H}_{2} \mathrm{O}$ $0.188 \mathrm{~g} \cdot \mathrm{L}^{-1} ; \mathrm{MgCl}_{2} \cdot 6 \mathrm{H}_{2} \mathrm{O} 1.083 \mathrm{~g} \cdot \mathrm{L}^{-1} ; \mathrm{CaCl}_{2} \cdot 2 \mathrm{H}_{2} \mathrm{O}$ $0.15 \mathrm{~g} \cdot \mathrm{L}^{-1} ; \mathrm{ZnSO}_{4}^{-} 7 \mathrm{H}_{2} \mathrm{O} 0.02 \mathrm{~g} \cdot \mathrm{L}^{-1} ;\left(\mathrm{NH}_{4}\right)_{2} \mathrm{SO}_{4} 0.063 \mathrm{~g} \cdot \mathrm{L}^{-1}$; $\mathrm{NH}_{4} \mathrm{Cl} 0.405 \mathrm{~g} \cdot \mathrm{L}^{-1}$ : yeast extract $1 \mathrm{~g} \cdot \mathrm{L}^{-1}$ and glycerol $30 \mathrm{~g} \cdot \mathrm{L}^{-1}$. OMP was adjusted to $\mathrm{pH} 5$ using dilute $\mathrm{HCl}$ and utilised without sterilisation.

M. pulcherrima was cultured in a fed-batch regime. The media were comprised of final effluent waste water; all nutrients other than yeast extract, biotin and the carbon source were supplied according to the OMP media. The media were not sterilised prior to use. Each culture was fed every 3 days up to 24 days $\left(25^{\circ} \mathrm{C}, 180 \mathrm{rpm}\right)$ and then allowed to rest at $15^{\circ} \mathrm{C}$ for 12 days without agitation, except phosphate, which was supplemented only at 15 days.

\section{Raceway pond cultivation}

OMP with a reduced glycerol content of $30 \mathrm{~g} \cdot \mathrm{L}^{-1}$ was used to establish cultures in two 500-L-capacity raceway ponds situated in a climate-controlled glasshouse. The ponds were inoculated with $500 \mathrm{ml}$ of $M$. pulcherrima culture grown for 48 hours at $25^{\circ} \mathrm{C}$ with agitation $(180 \mathrm{rpm})$ in yeast extract, mannitol and sorbose (YMS) medium consisting of $30 \mathrm{~g} \mathrm{~L}^{-1}$ yeast extract, $5 \mathrm{~g} \cdot \mathrm{L}^{-1}$ mannitol and $5 \mathrm{~g} \cdot \mathrm{L}^{-1}$ sorbose.

The pond cultures were agitated using a close-fitting paddle wheel driven at $10 \mathrm{rpm}$ and aerated through two spargers situated at opposite sides of the ponds. Culture temperature, $\mathrm{pH}$ and O.D.600nm were measured daily until the onset of the stationary phase, then every 4 days together with the addition of lipid fluorescence up to 28 days. With the onset of the stationary phase the temperature in the glasshouse was reduced from $25^{\circ} \mathrm{C}$ to $20^{\circ} \mathrm{C}$, the aeration was stopped and the paddle wheels were set at the minimum rotating rate $(4 \mathrm{rpm})$.

\section{Lipid extraction and analysis}

Lipid was extracted by an adaptation of a method first reported by Bligh and Dyer [40]. The samples were stirred in a $\mathrm{CHCl}_{3}$ and $\mathrm{MeOH}$ mixture $(2: 1 \mathrm{w} / \mathrm{v})$ over 48 hours, and the biomass was filtered off and washed with additional $\mathrm{CHCl}_{3}$. This was repeated three times. 
The volatiles were removed under reduced pressure and the lipid was analysed.

The lipid and sterol content were calculated by proton nuclear magnetic resonance $\left({ }^{1} \mathrm{H}\right.$ NMR) by comparison of the glyceride protons $(\delta 4.1 \mathrm{ppm})$ to the $\alpha$-protons of the sterol alcohol group ( $\delta 3.7$ to $3.9 \mathrm{ppm}$ ). NMR spectroscopic measurements were carried out at $298 \mathrm{~K}$ using a Bruker AV500 spectrometer (Bruker GmbH, Germany), operating at $300 \mathrm{MHz} .{ }^{1} \mathrm{H}$ spectra were typically acquired using a 30-degree excitation pulse and a repetition time of $4.2 \mathrm{sec} ; 0.3-\mathrm{Hz}$ line broadening was applied before Fourier transform, and spectra were referenced to the residual $\mathrm{CHCl}_{3}$ peak from the solvent $(\delta 7.26 \mathrm{ppm})$.

The glyceride proportion of the oil was transesterified using typical reported techniques [41]. The fatty acid profile was then calculated by gas chromatography-mass spectroscopy (GC-MS) with the resulting fatty acid methyl esters (FAME) component compared to known FAME standards. GC-MS analysis was carried out using an Agilent 7890A gas chromatograph equipped with a capillary column $(60 \mathrm{~m} \times 0.250 \mathrm{~mm}$ internal diameter $)$ coated with DB-23 ((50\%-cyanpropyl)-methylpolysiloxane) stationary phase $(0.25-\mu \mathrm{m}$ film thickness) and an He mobile phase (flow rate: $1.2 \mathrm{ml} /$ minute) coupled with an Agilent 5975C inert MSD with triple axis detector (Agilent Technologies, CA, USA). FAME samples were initially dissolved in $2 \mathrm{ml}$ of dioxane and $1 \mu \mathrm{l}$ of this solution was loaded onto the column, pre-heated to $150^{\circ} \mathrm{C}$. This temperature was held for 5 minutes and then heated to $250^{\circ} \mathrm{C}$ at a rate of $4^{\circ} \mathrm{C} /$ minute and then held for 2 minutes.

\section{Abbreviations \\ FAME: fatty acid methyl esters; GC-MS: gas chromatography-mass spectroscopy; NMR: nuclear magnetic resonance; O.D.: optical density; OMP: optimised M. pulcherrima; SMP: standard M. pulcherrima; YMD: yeast extract, malt extract and dextrose.}

\section{Competing interests}

A patent (UK No. 1302197.7) covering the majority of this work has been applied for. FS, RJS, CJC are the contributing authors.

\section{Authors' contributions \\ FS carried out the majority of the culturing including the open-tank reactor experiments. FW carried out a few of the culturing experiments and handled the extracting and analysis of the products produced. RS and CC conceived of the study, participated in its design and coordination and drafted the manuscript. All authors read and approved the final manuscript.}

\footnotetext{
Acknowledgements

The authors would like to extend their thanks to Graham Fisher and Miles Davis for their aid in securing funding, the Engineering and Physical Sciences Research Council (EPSRC) for partially funding this work through the Doctoral Training Centre at the Centre for Sustainable Chemical Technologies (CSCT), to Dr Marianne Harkins and Rhodri Jenkins for their help in harvesting the stirred tank reactors and to Roger and Sue Whorrod for their kind endowment to the University resulting in the Whorrod Fellowship in Sustainable Chemical Technologies held by Dr C Chuck.
}

Received: 29 July 2013 Accepted: 17 February 2014

Published: 4 March 2014

\section{References}

1. Department of Energy and Climate Change: UK renewable energy roadmap. London: Department of Energy and Climate Change; 2011:107.

2. Escobar JC, Lora ES, Venturini OJ, Yanez EE, Castillo EF, Almazan O: Biofuels: environment, technology and food security. Renew Sust Energ Rev 2009, 13:1275-1287.

3. Fargione J, Hill J, Tilman D, Polasky S, Hawthorne P: Land clearing and the biofuel carbon debt. Science 2008, 319:1235-1238.

4. Chisti Y: Biodiesel from microalgae beats bioethanol. Trends Biotechnol 2008, 26:126-131.

5. Sharma YC, Singh B, Korstad J: A critical review on recent methods used for economically viable and eco-friendly development of microalgae as a potential feedstock for synthesis of biodiesel. Green Chem 2011, 13:2993-3006.

6. Suali E, Sarbatly R: Conversion of microalgae to biofuel. Renew Sust Energ Rev 2012, 16:4316-4342.

7. Li Q, Du W, Liu D: Perspectives of microbial oils for biodiesel production. Appl Microbiol Biotechnol 2008, 80:749-756.

8. Yen H-W, Yang Y-C: The effects of irradiation and microfiltration on the cells growing and total lipids production in the cultivation of Rhodotorula glutinis. Biores Technol 2012, 107:539-541.

9. Tsigie YA, Lien Huong H, Ahmed IN, Ju Y-H: Maximizing biodiesel production from Yarrowia lipolytica Po1g biomass using subcritical water pretreatment. Biores Technol 2012, 111:201-207.

10. Huang C, Wu H, Liu Z-J, Cai J, Lou W-Y, Zong M-H: Effect of organic acids on the growth and lipid accumulation of oleaginous yeast Trichosporon fermentans. Biotechnol Biofuel 2012, 5:4.

11. Angerbauer C, Siebenhofer M, Mittelbach M, Guebitz GM: Conversion of sewage sludge into lipids by Lipomyces starkeyi for biodiesel production. Biores Technol 2008, 99:3051-3056.

12. Sun Y, Cheng JY: Hydrolysis of lignocellulosic materials for ethanol production: a review. Biores Technol 2002, 83:1-11.

13. Larsen J, Petersen MO, Thirup L, Li HW, Iversen FK: The IBUS process lignocellulosic bioethanol close to a commercial reality. Chem Eng Technol 2008, 31:765-772.

14. Koutinas AA, Chatzifragkou A, Kopsahelis N, Papanikolaou S, Kookos IK: Design and techno-economic evaluation of microbial oil production as a renewable resource for biodiesel and oleochemical production. Fuel 2014, 116:566-577.

15. Pulz O, Gross W: Valuable products from biotechnology of microalgae. Appl Microbiol Biotechnol 2004, 65:635-648.

16. Cripps RE, Eley K, Leak DJ, Rudd B, Taylor M, Todd M, Boakes S, Martin S, Atkinson T: Metabolic engineering of Geobacillus thermoglucosidasius for high yield ethanol production. Metab Eng 2009, 11:398-408.

17. Spadaro D, Vola R, Piano S, Gullino ML: Mechanisms of action and efficacy of four isolates of the yeast Metschnikowia pulcherrima active against postharvest pathogens on apples. Postharvest Biol Tech 2002, 24:123-134.

18. Kurtzman CP: The yeasts: A taxonomic study. 5th edition. Elsevier; 2011:2081

19. Fernandez M, Ubeda JF, Briones Al: Typing of non-saccharomyces yeasts with enzymatic activities of interest in wine-making. Int I Food Microbiol 2000, 59:29-36.

20. Kluyver AJ, Vanderwalt JP, Vantriet AJ: Pulcherrimin, the pigment of candida pulcherrima. Proc Natl Acad Sci U S A 1953, 39:583-593.

21. Roberts C: The effect of iron and other factors on the production of pigment by the yeast Torulopsis pulcherrima. Am J Bot 1946, 33:237-244.

22. Spadaro D, Ciavorella AA, Lopez-Reyes JG, Garibaldi A, Gullino ML: Effect of culture age, protectants, and initial cell concentration on viability of freeze-dried cells of Metschnikowia pulcherrima. Can J Microbiol 2010, 56:809-815

23. Fugelsang KL, Edwards CG: Wine Microbiology - Practical Applications and Procedures. 2nd edition. Springer; 2006:393

24. Jolly NP, Augustyn OPH, Pretorius IS: The role and use of non-saccharomyces yeasts in wine production. S Afr J Enol Vitic 2006, 27:15-39.

25. Rosini $G$, Federici $F$, Martini A: Yeast flora of grape berries during ripening. Microb Ecol 1982, 8:83-89.

26. Chatzifragkou A, Makri A, Belka A, Bellou S, Mavrou M, Mastoridou M, Mystrioti P, Onjaro G, Aggelis G, Papanikolaou S: Biotechnological conversions of biodiesel derived waste glycerol by yeast and fungal species. Energy 2011, 36:1097-1108.

27. Pitt JI, Miller MW: Sporulation in candida pulcherrima, candid reukaufii and chlamydozyma species - their relationships with Metschnikowia. Mycologia 1968, 60:663-685. 
28. Pan $L X$, Yang DF, Shao L, Li W, Chen GG, Liang ZQ: Isolation of the oleaginous yeasts from the soil and studies of their lipid-producing capacities. Food Technol Biotechnol 2009, 47:215-220.

29. Ratledge C, Wynn JP: The biochemistry and molecular biology of lipid accumulation in oleaginous microorganisms. In Advances in Applied Microbiology. 51st edition. Academic Press; 2002:1-51.

30. Charoenchai C, Fleet GH, Henschke PA: Effects of temperature, pH, and sugar concentration on the growth rates and cell biomass of wine yeasts. Am J Enol Viticult 1998, 49:283-288.

31. Meesters P, Huijberts GNM, Eggink G: High cell density cultivation of the lipid accumulating yeast Cryptococcus curvatus using glycerol as a carbon source. Appl Microbiol Biotechnol 1996, 45:575-579.

32. Liang Y, Cui Y, Trushenski J, Blackburn JW: Converting crude glycerol derived from yellow grease to lipids through yeast fermentation. Bioresour Technol 2010, 101:7581-7586.

33. Papanikolaou S, Fakas S, Fick M, Chevalot I, Galiotou-Panayotou M, Komaitis M, Marc I, Aggelis G: Biotechnological valorisation of raw glycerol discharged after bio-diesel (fatty acid methyl esters) manufacturing process: production of 1,3-propanediol, citric acid and single cell oil. Biomass Bioenergy 2008, 32:60-71.

34. Burgess JE, Quarmby J, Stephenson T: Role of micronutrients in activated sludge-based biotreatment of industrial effluents. Biotechnol Adv 1999, 17:49-70.

35. Fakas S, Galiotou-Panayotou M, Papanikolaou S, Komaitis M, Aggelis G: Compositional shifts in lipid fractions during lipid turnover in Cunninghamella echinulata. Enz Microb Technol 2007, 40:1321-1327.

36. Viikari L, Vehmaanpera J, Koivula A: Lignocellulosic ethanol: from science to industry. Biomass Bioenerg 2012, 46:13-24.

37. McMillan JD, Jennings EW, Mohagheghi A, Zuccarello M: Comparative performance of precommercial cellulases hydrolyzing pretreated corn stover. Biotechnol Biofuel 2011, 4:29.

38. Strauss MLA, Jolly NP, Lambrechts MG, Van Rensburg P: Screening for the production of extracellular hydrolytic enzymes by non-saccharomyces wine yeasts. J Appl Microbiol 2001, 91:182-190.

39. Chisti Y: Biodiesel from microalgae. Biotech Adv 2007, 25:294-306.

40. Bligh EG, Dyer WJ: A rapid method of total lipid extraction and purification. Can J Biochem Phys 1959, 37:911-917.

41. Freedman B, Butterfield R, Pryde E: Transesterification kinetics of soybean oil. J Am Oil Chem Soc 1986, 63:1375-1380.

doi:10.1186/1754-6834-7-34

Cite this article as: Santamauro et al:: Low-cost lipid production by an oleaginous yeast cultured in non-sterile conditions using model waste resources. Biotechnology for Biofuels 2014 7:34.

\section{Submit your next manuscript to BioMed Central and take full advantage of:}

- Convenient online submission

- Thorough peer review

- No space constraints or color figure charges

- Immediate publication on acceptance

- Inclusion in PubMed, CAS, Scopus and Google Scholar

- Research which is freely available for redistribution 